\title{
EL LIDERAZGO DEL DIRECTOR Y EL DESEMPEÑO DOCENTE AUTOPERCIBIDO EN UN GRUPO DE COLEGIOS PRIVADOS SALVADOREÑOS
}

\section{THE PRINCIPAL'S LEADERSHIP AND SELF-PERCEIVED TEACHING PERFORMANCE IN A GROUP OF SALVADORAN PRIVATE SCHOOLS}

\author{
Kenny Linette Orellana Hernández \\ Colegio Adventista de Quezaltepeque, El Salvador \\ kenny_nez@hotmail.com
}

\begin{abstract}
RESUMEN
La investigación -cuantitativa, descriptiva, correlacional y transversal-pretendió conocer si las dimensiones del liderazgo directivo -transformacional, transaccional y laissez-faire- predicen significativamente el desempeño docente, de acuerdo con la percepción de 105 docentes de un grupo de nueve colegios privados salvadoreños, quienes respondieron (a) el Cuestionario Multifactorial (MQL), de 45 items, y (b) el Cuestionario de Autoevaluación Docente, para medir la variable desempeño docente, de 20 items. Se utilizó el análisis de regresión múltiple. De las tres dimensiones del liderazgo del director, el liderazgo transaccional mostró una correlación positiva con el desempeño docente. La predicción es significativa para todas las dimensiones del desempeño docente, excepto emocionalidad. Los docentes que laboran en colegios cuyos directores están en función por más de siete años mostraron un desempeño significativamente mejor en las dimensiones de capacidad pedagógica y emocionalidad. A la vez, demostraron una percepción del liderazgo transformacional más baja en términos de motivación por inspiración.
\end{abstract}

Palabras clave: estilo de liderazgo, dirección escolar, desempeño docente

\section{ABSTRACT}

The research (quantitative, descriptive, correlational and cross-sectional) tried to discern if the dimensions of managerial leadership (transformational, transactional and 'laissez-faire') significantly predicted teaching performance based on the perception of 105 teachers from a group of nine private Salvadoran schools, who answered (a) the Multifactor Questionnaire (MQL), of 45 items, and (b) the Teacher Self-Assessment Questionnaire to measure the teaching performance variable of 20 items. Multiple regression analysis was used. Of the three dimensions of the principal's leadership, transactional leadership showed a positive correlation with teacher performance. The prediction is significant for all dimensions of teacher per- 


\section{ORELLANA HERNÁNDEZ}

formance, except emotionality. Teachers working in schools whose principals were in position for more than seven years showed significantly better performance in the dimensions of pedagogical capacity and emotionality. Nevertheless, they demonstrated a lower perception of transformational leadership in terms of motivation for inspiration.

Keywords: leadership style, school principal, teaching performance

\section{Introducción}

A lo largo de la historia de la humanidad han surgido hombres que se levantan a dirigir y guiar a un grupo, pueblo o nación. Hombres que con su visión, pasión, empuje y entrega, influyeron en las masas a tal punto de moverlas para alcanzar sus objetivos (Añazco Camacho, Valdivieso Salas y Sánchez Córdova, 2018).

Este estudio está basado primordialmente en la teoría de las relaciones, específicamente en los estilo de liderazgo transformacional y transaccional creados por Bass y Avolio. El liderazgo transformacional trabaja sobre las bases motivacionales de los empleados, llevándolos a apropiarse de la misión y visión de la empresa, a la autorrealización y al trabajo en beneficio del grupo, creando un verdadero compromiso con la empresa. Por otro lado, el liderazgo transaccional involucra un intercambio de intereses entre el empleador y el empleado. Ambos procuran beneficiarse del otro y se esfuerzan por logros personales. El liderazgo transaccional involucra premios o castigos como factores de motivación, vigilancia constante o libertad absoluta por parte del líder (Fernández y Quintero, 2017; Guanilo Pizarro, 2017).

En el área educativa, el director asume como máximo líder y es el encargado, junto con los docentes, de hacer transformaciones significativas en la mejora de la calidad académica y la integración de valores y principios en los estudiantes.

El propósito de esta investigación fue conocer las dimensiones de liderazgo - liderazgo transformacional, liderazgo transaccional y liderazgo laissez-faireque presentan en su quehacer laboral los nueve directores de los colegios adventistas seleccionados de la Unión Salvadoreña y verificar si existe una relación significativa entre las dimensiones de liderazgo y el desempeño ejercido por los docentes. Además, se quiso identificar si existen diferencias sobre los resultados entre los docentes de las instituciones adventistas que cuentan con un mismo director por más de siete años y los que tienen pocos años con el mismo director.

El objetivo de este estudio fue determinar si en las dimensiones de liderazgo directivo percibido por los docentes - liderazgo transformacional, liderazgo transaccional y liderazgo laissez-faire- son predictores significativos de la percepción de su propio desempeño académico —capacidad pedagógica, emocionalidad, responsabilidad en el desempeño de sus funciones laborales, relaciones interpersonales con sus alumnos y resultados de su labor educativaen nueve colegios privados de El Salvador durante el ciclo escolar 2018.

\section{Marco teórico \\ El liderazgo y sus estilos \\ El estilo de liderazgo se refiere a una serie de comportamientos relativa- mente duraderos en la forma de dirigir,}




\section{EL LIDERAZGO DEL DIRECTOR Y EL DESEMPEÑO DOCENTE}

que caracterizan al líder. Se han creado muchas teorías sobre los estilos de liderazgo a través del tiempo: teoría del gran hombre, teoría de los rasgos, teoría del comportamiento, teoría de la contingencia, teoría de la influencia y teoría de las relaciones (Giraldo González y Naranjo Agudelo, 2014).

Para este trabajo, se tomó en consideración la teoría de las relaciones de Bass y Avolio, que involucran los siguientes estilos de liderazgo: transformacional, transaccional y laissez-faire. Los estilos de liderazgo transformacional y transaccional constituyen dos estilos mutuamente opuestos. Como información complementaria, se incluyó la teoría de comportamiento, que involucra los estilos de liderazgo democrático, autoritario y laissez-faire.

\section{Liderazgo transaccional}

El líder transaccional negocia con los empleados lo que espera de ellos y el premio que obtendrán si alcanzan las metas. El premio está relacionado con el grado de cumplimiento de las metas. Se enfoca la relación del líder con su subalterno como en un tipo de juego donde se otorgan premios o castigos por una determinada labor, al estilo "garrote o zanahoria", es decir, existe una compensación positiva o negativa por los resultados alcanzados por los empleados (Reyes Gastañadui, 2018).

En este liderazgo basado en la relación de dar y recibir, el líder no pretende hacer cambios culturales sino que trabaja sobre la cultura organizacional establecida (Fernández y Quintero, 2017). La motivación está basada en el reforzamiento contingente (premios o castigos) de los empleados, lo cual genera interés pero de manera temporal y pasajera (Silva Peralta, Olsen, Pezzi y Sanjurjo, 2016).
El liderazgo transaccional presenta dos subescalas, que se describen a continuación:

Premio contingente: es la motivación basada en la entrega de premios o castigos. El líder transaccional genera una gama de premios o penalizaciones para el logro de sus objetivos (Rojas Jara, 2012).

Administración por excepción activo-pasivo: el líder monitorea el trabajo de su personal de forma activa o pasiva, durante el proceso o al final del mismo para responder a sus errores o aciertos

\section{Laissez-faire}

Se entiende el modelo laissez faire como la ausencia de liderazgo, de manera parcial o total. El líder laissez-faire se desenvuelve como quien "deja hacer" o "deja pasar" y delega las responsabilidades y la toma de decisiones a sus empleados, proporcionando poco apoyo y contacto.

Según variadas investigaciones, el liderazgo laissez-faire influye negativamente en el desempeño, la productividad y la innovación. Leal Soto, Albornoz Hernández y Rojas Parada (2016) observaron que la presencia del liderazgo pasivo-evitador se convierte en un obstáculo para la innovación de las instituciones educativas. Finalmente, Pacsi Choque, Estrada Mejía, Pérez Vásquez y Cruz Machaca (2015) mencionan que el liderazgo laissez faire influye negativamente en el desempeño de los trabajadores, pues se presenta una reducción en los resultados.

\section{Liderazgo transformacional}

El liderazgo transformacional, a diferencia del transaccional, trabaja con la motivación intrínseca de las personas. Se preocupa más por el ser que el 
hacer. Entre otras cosas desarrolla en los empleados autointerés, compromiso, autoconciencia, preocupación por el beneficio colectivo e identificación con la misión y la visión de la empresa, a fin de que todo el cuerpo de la empresa esté dispuesto a ir más allá de los que el trabajo exige para lograr un beneficio en conjunto (Mendoza Torres y Ortiz Riaga, 2006). El líder transformacional se distingue por cuatro características básicas: (a) la influencia idealizada, (b) la motivación inspiracional, (c) la estimulación intelectual y (d) la consideración individual.

Influencia idealizada (carisma). Un líder carismático posee capacidades peculiares y su suspicaz personalidad lo distingue entre los demás, por lo que es admirado, respetado e imitado por los miembros del grupo. El líder carismático es considerado por sus subalternos como poseedor de un alto grado de moralidad, confianza e integridad. Además, transmite entusiasmo, respeto al personal, confianza, seguridad, lealtad y compromiso, obteniendo la total colaboración del personal para los logros óptimos de desarrollo y producción (Cervera Cajo, 2012). El líder carismático influye significativamente en el desempeño de sus subordinados.

Motivación inspiradora. La motivación que el líder transmite a los empleados influye positivamente en el desempeño laboral, pues el trabajo se realiza con entusiasmo y satisfacción (Reynaga Utani, 2015; Sum Mazariegos, 2015). Por otro lado, Pérez Perea, Soler Cárdenas y Díaz Hernández (2009) observaron que la falta de motivación produce un trabajo mecánico, automatizado, un inadecuado ambiente laboral y finalmente un servicio deficiente y con poca calidad.
Estimulo intelectual. El líder estimula al personal para que propongan nuevas soluciones, a hacerse preguntas, a generar nuevas ideas, dándoles libertad de expresarse sin coartarlos. Según Arana Agüero y Coronado Tarrillo (2017), el líder transformacional es generador de cambio, delega responsabilidades y da autonomía a los subalternos acompañándolos en su ejercicio laboral.

González, González, Ríos y León (2013) observaron que los docentes universitarios que practicaban la estimulación intelectual en su quehacer educativo animaban a los estudiantes a proponer nuevas soluciones a antiguos problemas, promovían la reflexión, la criticidad y la racionalidad, desarrollaban la creatividad y la innovación en su desempeño, lo cual les permitía optimizar su desempeño y mejorar la calidad del proceso de enseñanza aprendizaje.

Consideración individual. El líder mantiene una relación personal con cada empleado, conoce sus necesidades y anhelos, lo escucha, le ayuda a superarse y a reafirmar su autoestima y le provee de retos y oportunidades que desarrollan su confianza y seguridad para que pueda proyectarse a responsabilidades cada vez superiores (Cervera Cajo, 2012; Chacón Luna, 2016; Minaya Canales, 2014).

Llorens, Salanova y Losilla (2009) observaron que un líder transformador genera afectos positivos en sus empleados tales como entusiasmo, optimismo, satisfacción y otros estados emocionales positivos más estables. Perilla Toro y Gómez Ortiz (2017) observaron que el liderazgo transformacional tiene una relación negativa con los síntomas de malestar, es decir, que un líder transformacional genera menor malestar (en aspectos afectivos) en los empleados. 


\section{EL LIDERAZGO DEL DIRECTOR Y EL DESEMPEÑO DOCENTE}

\section{Investigaciones empíricas}

Las investigaciones sobre los estilos de liderazgo transformacional y transaccional indican una correlación fuerte y positiva entre todas las dimensiones de liderazgo transformacional y las mediciones objetivas y subjetivas de desempeño laboral (Arana Agüero y Coronado Tarillo, 2017; Cesar Rivera, 2018; Cruz Ortiz, Salanova Soria y Martínez Martínez, 2013; Espinoza Poves, 2017; García Rojas, 2017; Onorato, 2013; Salas Vallina, 2013). La dimensión de recompensa contingente también mostró una relación positiva pero menos fuerte; por otra parte, la dirección por excepción pasiva fue correlacionada negativamente con el desempeño (Lowe, Kroeck y Sivasubramaniam, 1996).

También el liderazgo transformacional tiene un impacto significativo sobre la satisfacción laboral y el compromiso organizacional, lo que conlleva a un buen desempeño laboral. En variadas investigaciones (Aydin, Sarier y Uysal, 2013; Chacón Luna, 2016; Félix Román, 2014; Minaya Canales, 2014; Yang, 2012) los resultados mostraron que el estilo de liderazgo transformacional afecta positivamente a la satisfacción laboral y el compromiso organizacional de los profesionales. Y que los administradores que cambiaron del estilo de liderazgo transaccional al transformacional vieron elevado los niveles de satisfacción laboral y de compromiso organizacional de los docentes.

El liderazgo transformacional tienen un impacto positivo sobre la eficacia laboral y la calidad de los colegios, a diferencia del liderazgo transaccional y el laissez faire (Hermosilla, Amutio, Costa y Páez, 2016; Huillca Condori, 2015; Pedraja Rejas, Rodríguez Ponce, Barreda Olavarría, Sagredo Núñez y Segovia
León, 2009; Pedraja Rejas, Rodríguez Ponce, Delgado Almonte y Rodríguez Ponce, 2006). Vale la pena mencionar que el liderazgo transformacional del director tiene un largo alcance, pues influye positivamente en los logros académicos de los estudiantes (Pedraja Rejas, Rodríguez Ponce y Rodríguez Mardones, 2016).

Por otro lado, la falta de liderazgo transformacional en el director se refleja en el bajo desempeño del docente. Parra Rivas (2011) hizo una investigación en escuelas bolivianas y encontró que los docentes evaluaron a sus directores con un criterio bajo en las siguientes áreas: liderazgo transformacional, funciones y perfil del director. A la vez, los docentes se autoevaluaron con un criterio bajo en las siguientes áreas: características del docente en el aula y roles como docentes. Con todo, algunas investigaciones presentan lo contrario. Bravo Salinas y Vicente Acevedo (2013) encontraron, en un hospital público, que no existía una relación significativa entre el liderazgo transformacional y el desempeño laboral; pero la verdad es que la mayoría de las investigaciones en el campo educativo encuentran una relación significativa entre el liderazgo transformacional y el desempeño laboral.

Los docentes que ejercen un liderazgo transformacional en sus aulas consiguen un mejor desempeño laboral y por ende elevan el rendimiento académico en los estudiantes. González et al. (2013) mencionan que todos los atributos del liderazgo transformacional, tales como consideración individual, motivación inspiracional, estimulación intelectual e influencia idealizada, presentes en la labor diaria del docente universitario, permitirán optimizar su propio desempeño laboral y por ende mejorar la calidad 
de la enseñanza y el aprendizaje. Según Ramón Molina, Muñoz Aparicio, Ancona Alcocer y Navarrete Torres (2015), el tipo de perfil de liderazgo que más impacta sobre el aprendizaje y el rendimiento de los estudiantes universitarios es el democrático transformacional.

\section{Liderazgo según la teoría del comportamiento}

Quispe Quispe (2011) menciona que los estilos de liderazgo democrático, autocrático y liberal están basados en el comportamiento del líder sin considerar las características de personalidad. Explican cómo se comporta el líder respecto a sus seguidores y cómo logra liderarlos. A continuación se describen brevemente.

Estilo autocrático. El estilo autocrático se basa en el cumplimiento rápido y efectivo de órdenes y en el alcance de objetivos. El líder ejerce su autoridad, se coloca en una posición de superioridad, toma decisiones sin consultar al cuerpo de empleados y establece metas que deben ser aceptadas y alcanzadas por los métodos que él indique. Este liderazgo fomenta relaciones de miedo, pasividad y desconfianza, pues el líder no estimula, ni incentiva, ni apoya a los miembros del grupo, pero elogia o critica el trabajo individual (Pazmiño Solys, Beltrán Morales y Gallardo Media, 2016; Ponce Vidal, 2008; Quispe Quispe, 2011).

Estilo democrático o participativo. El liderazgo democrático está relacionado con el buen desempeño laboral. El líder democrático motiva a la participación del grupo, está atento a las ideas de los demás y comparte con libertad las suyas. Se coloca en una situación de iguales con todo el equipo y fomenta relaciones de confianza, democracia, amistad y diálogo. Se preocupa por el desarrollo individual de los empleados y sus necesidades, por la existencia de relaciones interpersonales sanas y por la integración de todo el personal como un solo equipo. Las metas son alcanzadas por un trabajo concienzudo, colaborativo, voluntario y comprometido (Pazmiño Solys et al., 2016; Quispe Quispe, 2011; Zarate Ramírez, 2011).

Estilo liberal o permisivo. El liderazgo liberal está relacionado con un mal desempeño laboral. Según Pacsi Choque et al. (2015), el liderazgo liberal influye negativamente en el desempeño laboral de los empleados, pues no se alcanzan las metas y el producto es de baja calidad. Existe libertad completa para el grupo o en las decisiones individuales. El líder se sitúa al margen, muestra desinterés, desatiende sus responsabilidades y al grupo, tiene una mínima participación, no toma parte en la evaluación del trabajo realizado, evita interferir y deja que todo tome su propio curso. Un líder liberal es permisivo o incapaz de dirigir a un grupo de personas (Quispe Quispe, 2011; Zarate Ramírez, 2011).

\section{Desempeño del docente: el docente y sus roles}

Concepto de desempeño. El desempeño son acciones o comportamientos que los empleados presentan y que influyen directamente en el logro de los objetivos de la organización (Chiavenato, 2002).

Según Chiang Vega, Méndez Urra y Sánchez Bernales (2010), el desempeño mide la cantidad y calidad del trabajo, el grado de cooperación y responsabilidad, la asistencia, la disponibilidad de evaluación, el dominio del trabajo y principalmente la eficiencia en el logro de los objetivos mientras se usan adecuadamente los recursos. 


\section{EL LIDERAZGO DEL DIRECTOR Y EL DESEMPEÑO DOCENTE}

El desempeño está enfocado en la labor cotidiana del individuo que involucra el desarrollo de sus funciones, deberes y responsabilidades inherentes a su trabajo, la forma y los resultados que obtiene, a la vez que implica una interrelación de competencias, actitudes y valores (Alonso Ayala, Ávila Sánchez y Sánchez López, 2016).

Desempeño del docente. Es el cumplimiento de sus funciones o responsabilidades las cuales están asociadas al propio docente, los estudiantes y la institución. El desempeño docente se ve manifiesto en variados niveles: el contexto socio-cultural, el ambiente escolar, las vivencias en el aula y sobre el propio docente (Zarate Ramírez, 2011).

El docente debe cumplir con diversas actividades en variados ambientes y momentos. Los roles de los docentes se enfocan en las siguientes áreas: (a) profesional, (b) personal y (c) social.

Dimensión profesional. Como profesional, el docente planifica el proceso educativo adecuándose a las necesidades del grupo, usa variadas estrategias para asegurarse el aprendizaje y evalúa sin que los estudiantes se intimiden; también fomenta un clima de respeto, compañerismo y participación en el aula (Zarate Ramírez, 2011). El docente debe preparar las clases con dedicación, responsabilidad y eficiencia, a la vez que ser claro y conciso en la exposición del contenido, exigente y equitativo al evaluar (Sgreccia y Cirell, 2015).

El esfuerzo concienzudo que se hace para desarrollar una práctica docente organizada y sistematizada genera buenos resultados en los estudiantes y en la institución educativa (Martínez, 2015).

Dimensión personal. En el área personal, el docente se muestra como un ser intelectual, crítico, creativo, innovador y adaptable al medio. Desarrolla una sana autoestima y una personalidad equilibrada con principios y valores definidos, actuando proporcionalmente a lo que habla y espera de los estudiantes, cultiva un espíritu de servicio y se muestra accesible a quienes lo necesitan (Zarate Ramírez, 2011).

Según Martínez de la Hidalga y Villardón Gallego (2015), entre las cualidades con las que debe contar un buen profesor de secundaria, están el dominio de su rama académica, la motivación, la innovación, las buenas relaciones sociales, la promoción de la convivencia en el aula, el disfrute de su trabajo, la amabilidad, la empatía, la comprensión, la disciplina dentro del aula, la paciencia y la responsabilidad.

Dimensión social. En el área social, el docente prepara hombres con valores y principios que se conviertan en líderes, por lo cual procura ser ejemplo de trabajo y perseverancia. Se identifica con los proyectos de la comunidad, muestra su apoyo en la solución de problemas que se presentan y refuerza la identidad nacional, fomentando actividades culturales, recreativas y deportivas, procurando vivir como un verdadero agente de cambio (Zarate Ramírez, 2011).

\section{Conceptualizaciones de las dimensiones de nivel de desempeño docente}

Para efectos de esta investigación, el nivel de desempeño docente será medido por las siguientes dimensiones (Quispe Quispe, 2011): (a) capacidad pedagógica: dominio de los contenidos en su rama de especialidad, estilo de planificación, desarrollo de la clase, metodologías y aplicación del conocimiento en la vida práctica; (b) emocionalidad: dimensión que involucra la 


\section{ORELLANA HERNÁNDEZ}

personalidad del docente, su vocación, autoestima, satisfacción y seguridad al desarrollar sus clases; (c) responsabilidad en el desempeño de sus funciones laborales: grado de entrega del docente a la institución, puntualidad, asistencia, cumplimiento de deberes y debida atención a estudiantes; (d) relaciones interpersonales con sus alumnos: interés que el docente muestra en los estudiantes y en sus necesidades individuales -físicas, mentales, emocionales y espirituales-, tanto dentro como fuera de la escuela; y (e) resultado de su labor educativa: impacto y transcendencia de la enseñanza del docente manifestado en las competencias alcanzadas por los estudiantes, promedio general de su materia y nivel de competencias alcanzadas en relación al estándar del país.

\section{Expectativas del alumno respecto del buen profesor}

Según Fernández y Álvarez (2002, citados en Zarate Ramírez, 2011), para todo estudiante independientemente de sus logros académicos, un buen profesor cumple con las siguientes características:

1. Claridad en las explicaciones: expresan claramente sus ideas y son comprendidos incluso por aquellos estudiantes que muestran un mínimo interés.

2. Entusiasmo en su trabajo: disfrutan y dominan su materia y contagian al grupo con su entusiasmo.

3. Motivación: valoran el trabajo y el esfuerzo de sus alumnos, elogiando sus logros aunque estos sean pequeños. Ayudan a los estudiantes a organizarse y responsabilizarse de sus trabajos.

4. Interacción: se relacionan con sus alumnos por medio de conversaciones, juegos y otras actividades que no necesariamente involucren temas académicos.
5. Autoridad: dominan el grupo sin ser necesariamente duros ni inflexibles, generando un clima adecuado para el aprendizaje.

De manera muy similar, Sgreccia y Cirelli (2015) observaron cinco dimensiones que los alumnos destacan como principales para identificar a un buen profesor: motivación estudiantil (que el docente disfrute lo que hace y lo contagie al grupo), una buena preparación de la clase, la gestión de la clase (incluye la forma de explicar la clase y la disciplina en el aula), el trato con los alumnos y el proceso de evaluación (exigencias del profesor).

\section{Relación entre el liderazgo del director y el desempeño docente}

Existe una relación significativa entre el estilo de liderazgo del director y el desempeño del docente (Aguilar Ludeña, 2018; Ayvar Bazán, 2014; Julca Chuquista, 2014; Mora Acuña, 2017; Ortiz Chávez, 2016; Ponce Luque, 2018; Quispe Quispe, 2011). El desempeño del docente se ve afectado positiva o negativamente dependiendo del liderazgo que asuma el director. Se puede precisar que el nivel de desempeño docente depende mucho del estilo de liderazgo del director; es decir, a mayores valores en la aplicación de un buen estilo de liderazgo del director se obtienen mayores valores en el nivel de desempeño de los docentes y viceversa. Además, según Ramos Cuba (2015), existe una correlación positiva moderada entre el liderazgo del director y la planificación del trabajo curricular, el clima del aula y la planificación del trabajo curricular docente. También existe una correlación positiva próxima a la media entre el liderazgo del director y la conducción del proceso de enseñanza-aprendizaje. 


\section{Metodología \\ Tipo de investigación}

Esta investigación utilizó un abordaje cuantitativo, transversal, descriptivo y correlacional.

\section{Participantes}

La muestra para esta investigación estuvo conformada por 105 de los 125 docentes que laboran en los nueve colegios privados seleccionados de el Salva- dor, durante el período escolar 2018. Se presenta en la Tabla 1 la distribución de los participantes por colegio.

\section{Instrumentos}

Medición del liderazgo del director. El instrumento utilizado para el estudio de la variable liderazgo del director fue una adaptación del Cuestionario Multifactorial sobre Liderazgo, segunda edición, versión corta, conocido por sus

Tabla 1

Distribución de docentes por colegios

\begin{tabular}{lccc}
\hline \multicolumn{1}{c}{ Institución } & $\begin{array}{c}\text { Total de } \\
\text { docentes }\end{array}$ & Participantes & $\%$ \\
\hline Colegio Adventista de Chalatenango & 12 & 10 & 9.52 \\
Colegio Adventista de Santa Ana & 18 & 15 & 14.29 \\
Colegio Adventista Central de San Salvador & 13 & 12 & 11.43 \\
Colegio Adventista de Ayutuxtepeque & 14 & 10 & 9.52 \\
Colegio Adventista de Quezaltepeque & 18 & 16 & 15.24 \\
Escuela de Capacitación Adventista & 24 & 19 & 18.10 \\
Colegio Adventista de Santa Tecla & 8 & 8 & 7.62 \\
Colegio Adventista de Soyapango & 13 & 10 & 9.52 \\
Colegio Adventista de Citalá & 5 & 5 & 4.76 \\
Total & 125 & 105 & 100 \\
\hline
\end{tabular}

siglas en inglés $M L Q$ (Form $5 X$-Short). Creado por los investigadores estadounidenses Bass y Avolio en el año 2000, con un formato para el docente compuesto por 45 ítems (Rojas Jara, 2012).

Para calcular la confiabilidad de esta versión adaptada del cuestionario, Rojas Jara (2012) administró el instrumento a una muestra de 20 docentes. Los resultados arrojaron un alfa de Cronbach de .836 , mostrando una alta confiabilidad.

El Cuestionario Multifactorial está estructurado para el análisis de tres grandes ejes: (a) liderazgo transformacional, (b) liderazgo transaccional y (c) lais- sez-faire. En el cuestionario se encuentran preguntas relacionadas para cada eje de manera aleatoria y los resultados se obtienen por los puntajes alcanzados en cada dimensión (Rojas Jara, 2012). La Tabla 2 presenta una síntesis de la información.

El instrumento mide otras dimensiones (satisfacción, esfuerzo extra y eficacia) que no fueron consideradas en este estudio por razones de utilidad.

Medición del desempeño del docente. El cuestionario para el estudio de la variable desempeño docente fue elaborado por Quispe Quispe (2011). Está constituido por 20 ítems distribuidos en 
cinco dimensiones de la siguiente manera: capacidad pedagógica, cinco ítems (1, 2, 3, 4 y 5); emocionalidad, cuatro ítems (6, 7, 8 y 9); responsabilidad en el desempeño de sus funciones laborales, cuatro ítems (10, 11, 12 y 13); relaciones interpersonales con sus alumnos, cuatro ítems $(14,15,16$ y 17) y resultados de su labor educativa, tres ítems $(18,19$ y 20).

Para obtener la validez del instrumento administrado a los docentes, Quispe Quispe (2011) lo sometió a una prueba piloto de 21 docentes de la muestra y obtuvo un alfa de Cronbach de .822 , considerándose un indicador de alta confiabilidad.

\section{Análisis de datos}

Se recurrió a la estadística descriptiva para evaluar el comportamiento de las variables y poner a prueba la hipótesis. Se utilizó el análisis de correlación y el análisis de regresión múltiple.

Las variables del estudio fueron las prácticas de liderazgo del director y el desempeño docente autopercibido.

\section{Resultados}

Para determinar si las dimensiones de liderazgo directivo percibido por los docentes - liderazgo transformacional, liderazgo transaccional y liderazgo laissez-faire- eran predictoras significativas de la percepción de su propio desempeño laboral en nueve colegios privados de El Salvador durante el ciclo escolar 2018, se utilizó la regresión lineal múltiple.

Se observa que el modelo compuesto por las variables liderazgo laissez-faire, liderazgo transformacional y liderazgo transaccional explica el $10.4 \%$ de la variable desempeño laboral autopercibido. Es decir, el coeficiente de determinación indica que el $10.4 \%$ de la variación del desempeño docente está explicada por el estilo de liderazgo del director. Los resultados arrojados de la prueba computarizada demuestran que hay suficiente evidencia para rechazar el enunciado $\left(F_{(3,101)}=3.899, p=.011, R=.322, R^{2}=\right.$ $.104, R^{2}$ corregida $\left.=.077\right)$.

Como el aporte del liderazgo laissez faire no resulta significativo, el mejor modelo predictor está formado por las dimensiones liderazgo transaccional y liderazgo transformacional $\left(F_{(2,102)}=\right.$ $5.761, p=.004, R=.319, R^{2}=.102, R^{2}$ corregida $=.084)$. Al observar el tamaño de los coeficientes estandarizados beta, es importante considerar que el único aporte estadísticamente significativo es el del liderazgo transaccional $(\beta=.607$, $p=.006$ ).

\section{Correlaciones entre las dimensiones de las variables}

\section{Liderazgo transformacional $\mathbf{y}$} desempeño docente. A continuación se presentan las correlaciones de la dimensión del liderazgo transformacional y sus subdimensiones con el desempeño docente y sus dimensiones.

Existe una correlación positiva débil, aunque significativa, entre el liderazgo transformacional y la dimensión responsabilidad del docente $(r=.200$, $p=.041$ ). Igualmente se observa una correlación positiva débil, aunque significativa, entre la subdimensión motivación por inspiración y la dimensión relaciones interpersonales del docente $(r$ $=.194, p=.048$ ).

Finalmente, es muy importante considerar que las dimensiones responsabilidad, relaciones interpersonales y resultados de la labor educativa muestran correlaciones positivas con la subdimensión consideración individual por parte del director. Es decir, una mayor consideración individual por parte del 


\section{EL LIDERAZGO DEL DIRECTOR Y EL DESEMPEÑO DOCENTE}

director indica un mejor desempeño del docente (ver Tabla 2).

Liderazgo transaccional y desempeño docente. La Tabla 3 presenta las correlaciones significativas existentes del liderazgo transaccional y sus subdimensiones con el desempeño docente y sus dimensiones.

Existe suficiente evidencia estadística para afirmar que el liderazgo transaccional se relaciona significativamente con el desempeño docente. La única dimensión del desempeño docente que no se correlaciona significativamente con el liderazgo transaccional es la emocionalidad (liderazgo transaccional, $r=.097, p=.323$; recompensa contingente, $r=.123, p$ $=.212$; dirección por excepción, $r=$ $.052, p=.601)$.

\section{Tabla 2}

Correlaciones significativas entre la consideración individual y el desempeño docente y sus dimensiones

\begin{tabular}{|c|c|c|}
\hline \multirow[b]{2}{*}{ Variable/dimensión } & \multicolumn{2}{|c|}{ Consideración individual } \\
\hline & $r$ & $p$ \\
\hline Desempeño docente & .211 & .031 \\
\hline Responsabilidad & .241 & .013 \\
\hline Relaciones interpersonales & .217 & .026 \\
\hline Resultados de labor educativa & .223 & .022 \\
\hline
\end{tabular}

Como se puede observar, los valores del coeficiente de correlación $r$ de Pearson entre las variables del estudio y entre sus dimensiones se mantiene entre .206 y .327 . Estos valores representan relaciones positivas más bien débiles.

En conclusión, el liderazgo transaccional y sus subdimensiones - recompensa contingente y dirección por excepciónmantienen una correlación positiva con la mayoría de las dimensiones del desempeño docente. La única dimensión que no correlaciona es la emocionalidad.

\section{Otros análisis}

Uno de los objetivos de esta investigación fue comparar los resultados entre las instituciones que cuentan con el mismo director por siete o más años y aquellas cuyos directores tienen seis años o menos en su función.

La prueba $t$ de Student para grupos independientes arrojó resultados que se indican a continuación. Los docentes en cuyos colegios el director tiene pocos años en función muestran una media significativamente mayor en la subdimensión motivación por inspiración del liderazgo transformacional $(M=4.2241$, $D E=.82941)\left(t_{(87,705)}=-2,129, p=.036\right)$, en comparación con los docentes en cuyo colegio el director cuenta con pocos años en el ejercicio de su función ( $M$ $=3.8298, D E=1.02696$ ).

Los docentes de los colegios que cuentan con el mismo director por muchos años muestran una media significativamente mayor en la dimensión capacidad pedagógica de la variable desempeño docente $(M=4.2000, D E$ $=.46062)\left(t_{(97.450)}=2.199, p=.030\right)$, en comparación con los docentes de los colegios que tienen pocos años con el mismo director $(M=4.0034, D E=.44877)$. 


\section{ORELLANA HERNÁNDEZ}

Finalmente, los docentes cuyos colegios tienen siete o más años con el mismo director a cargo muestran una media significativamente mayor en la dimensión emocionalidad de la variable desempeño docente $(M=4.5213, D E=$
.41972) $\left(t_{(101.966)}=2.613, p=.010\right)$, en comparación con los docentes que cuentan con pocos años con el mismo director $(M=4.2672, D E=.57519)$.

Tabla 3

Correlaciones significativas entre el

liderazgo transaccional y el desempeño docente y sus respectivas dimensiones

\begin{tabular}{lccccccc}
\hline & \multicolumn{2}{c}{$\begin{array}{c}\text { Liderazgo tran- } \\
\text { saccional }\end{array}$} & \multicolumn{2}{c}{$\begin{array}{c}\text { Recompensa } \\
\text { contingente }\end{array}$} & \multicolumn{2}{c}{$\begin{array}{c}\text { Dirección por } \\
\text { excepción }\end{array}$} \\
\cline { 2 - 8 } \multicolumn{1}{c}{ Variables/ dimensiones } & $r$ & $p$ & $r$ & $p$ & $r$ & $p$ \\
\hline Desempeño docente & .274 & .005 & .284 & .003 & .217 & .026 \\
Responsabilidad & .281 & .004 & .327 & .001 & & \\
Capacidad pedagógica & .206 & .035 & & & .229 & .019 \\
Relaciones interpersonales & .279 & .004 & .287 & .003 & & \\
Resultados de labor educativa & .264 & .006 & .274 & .005 & .209 & .032 \\
\hline
\end{tabular}

\section{Discusión}

El estudio reportó para las dimensiones de la variable liderazgo directivo y la variable desempeño docente los siguientes valores de la media y desviación estándar. Para el liderazgo transformacional, se obtuvo una media igual a $3.8976(D E=.81634)$; para el liderazgo transaccional, una media igual a 3.7238 $(D E=.81392)$; para el liderazgo laissez-faire, una media igual a $2.3286(D E$ $=.77294$ ) y para la variable del desempeño docente, una media igual a 4.1924 $(D E=.43885)$, en un rango posible de 1 a 5 puntos.

Se observó una predicción significativa de la percepción del desempeño docente a partir de la dimensión liderazgo transaccional, sobre la base de una correlación positiva. También se observó que la subdimensión consideración individual, del liderazgo transformacional, está correlacionada positivamente con las siguientes dimensiones del desempeño docente: responsabilidad, relaciones interpersonales y resultados de la labor educativa.
Según los resultados, todas las dimensiones del desempeño docente, menos la dimensión de emocionalidad -satisfacción, autoestima, valoración y seguridad-, mantienen una correlación positiva con la dimensión liderazgo transaccional.

Finalmente, al comparar los resultados obtenidos entre los docentes de los colegios que cuentan con un mismo director por siete o más años y los de los docentes cuyos colegios tienen el mismo director por pocos años, se encontraron diferencias significativas mayores en las dimensiones capacidad pedagógica y emocionalidad del desempeño docente, a favor de los docentes cuyos colegios cuentan con el mismo director por muchos años. Se observó también una diferencia significativa mayor en la subdimensión motivación por inspiración del liderazgo transformacional, esta vez en favor de los docentes de colegios cuyos directores tienen pocos años en función.

Los hallazgos encontrados permiten aceptar que las dimensiones de liderazgo 


\section{EL LIDERAZGO DEL DIRECTOR Y EL DESEMPEÑO DOCENTE}

percibido por los docentes - liderazgo transformacional, liderazgo transaccional y liderazgo laissez-faire- son predictores significativos de la percepción de su propio desempeño académico, en nueve colegios adventistas de El Salvador durante el ciclo escolar 2018.

El análisis de los resultados indica que, de las tres dimensiones de la variable independiente liderazgo directivo, únicamente la dimensión liderazgo transaccional aporta significativamente al desempeño docente. Estos resultados coinciden con otros estudios que afirman que el liderazgo transaccional está relacionado con el desempeño (Coronado Quintana, Domínguez Canizalez, Olivares Leal y Retes López, 2014; Reyes Gastañadui, 2018; Rodríguez Ponce, Pedraja Rejas y Ganga Contreras, 2017).

El liderazgo transaccional está basado en el intercambio de valores entre el líder y sus empleados. El líder ofrece premios o castigos económicos, psicológicos, políticos u otros como motivación para que el empleado logre sus objetivos. A mayores logros, ofrece mayores premios y, viceversa, a mayores fracasos ofrece mayores castigos. Cada parte, líder y empleados, van en busca de su propio beneficio (Añazco Camacho et al., 2018).

Los resultados de esta investigación indican que la dimensión liderazgo transaccional se relaciona significativamente con cada una de las dimensiones del desempeño docente, excepto con la dimensión emocionalidad - personalidad, vocación, autoestima, satisfacción y seguridad al impartir las clases-. Este resultado no resulta extraño, pues algunas investigaciones hallaron que el liderazgo transaccional guarda una relación negativa con la satisfacción laboral, ya que se le da mayor importancia al logro de los objetivos que al bienestar del personal (Añazco Camacho et al., 2018; Salem, 2015). Lo ideal para generar entusiasmo, optimismo, tranquilidad, valoración, satisfacción, compromiso y en general estados emocionales positivos estables y prolongados en el personal, es que el líder pase de transaccional a transformacional (Alzate Sánchez y López Cortés, 2014; Aydin et al., 2013; Llorens et al., 2009).

Basados en los resultados de esta investigación, se puede inferir que los directores mayormente motivan al personal por intercambios de premios o castigos a fin de alcanzar sus objetivos o proyecciones. Con todo, el premiar por el alcance de los objetivos no es del todo negativo. Lo que ocurre es que no siempre existen los recursos para hacerlo. En cuanto a los castigos, no todos los docentes los toman de una manera positiva. Lo que en realidad preocupa es el hecho de que la emocionalidad de los docentes está siendo golpeada. Los docentes cumplen con las actividades que su profesión exige, pero se afectan su autoestima, su valoración y su satisfacción, lo cual, con el tiempo, influirá negativamente sobre la calidad de su desempeño académico y sobre el cumplimiento de la misión que asumen como instituciones.

Los resultados también indicaron que los directores que incluyen en su práctica de liderazgo la consideración individual — subdimensión del liderazgo transformacional - ven elevado el desempeño laboral de sus docentes. Esto coincide con el estudio de Chen (2013), donde afirma que la preocupación empática hacia los profesores y su cuidado, de parte del director, eleva su desempeño académico. Y que el liderazgo que toma como base las emociones de los empleados asegura un mejor desempeño laboral. 


\section{ORELLANA HERNÁNDEZ}

Finalmente, los resultados muestran que se encontraron medias significativas mayores en las dimensiones capacidad pedagógica y emocionalidad del desempeño docente, en los docentes de colegios que cuentan con el mismo director por muchos años. A la vez, se les observó una media significativa menor en la subdimensión motivación por inspiración, del liderazgo transformacional. Estos datos indicarían que el grupo de docentes que tiene muchos años trabajando con el mismo director adquiere y afianza sus habilidades en el ejercicio de su práctica profesional y maneja una mejor emocionalidad, pues entre otros aspectos, no se enfrenta con la resistencia al cambio y la aceptación de un nuevo director. Por otra parte, los directores que se perpetúan en el puesto van perdiendo la capacidad de motivar e inspirar a su personal.

\section{Referencias}

Aguilar Ludeña, E. H. (2018). Liderazgo directivo y el desempeño docente en la institución educativa 1278, La Molina (Tesis de maestría). Universidad Nacional de Educación Enrique Guzmán y Valle, Perú.

Alonso Ayala, O., Ávila Sánchez, M. y Sánchez López, M. (2016). Desempeño del profesional de enfermería en la atención a los pacientes con afecciones traumatológicas y ortopédicas. $R e$ vista Cubana de Tecnología de la Salud, 7(4), 30-35.

Alzate Sánchez, A. y López Cortés, M. C. (2014). Impacto del liderazgo transformacional y la felicidad en la cultura organizacional - caso Google informe del VII seminario internacional en gestión de las organizaciones Estados Unidos 2014 (Trabajo de grado). Universidad de Bogotá Jorge Tadeo Lozano, Bogotá, Colombia.

Añazco Camacho, K. A., Valdivieso Salas, R. P. y Sánchez Córdova, S. W. (2018). Los estilos de liderazgo y su efecto en la satisfacción laboral. INNOVA, 3(10), 142-148.

Arana Agüero, L. y Coronado Tarrillo, J. M. (2017). Liderazgo directivo y desempeño docente en una institución educativa parroquial del distri- to de San Isidro (Tesis de maestría). Universidad Marcelino Champagnat, Lima, Perú.

Aydin, A., Sarier, Y. y Uysal S. (2013). The effect of school principals' leadership styles on teachers' organizational commitment and job satisfaction". Educational Sciences: Theory \& Practice, 13(2), 806-811.

Ayvar Bazán, Z. (2014). Liderazgo pedagógico del director y evaluación del desempeño docente en las instituciones educativas del nivel secundario de la red $\mathrm{n}^{\circ} 09$ del distrito de Villa María del Triunfo. LOGOS, 6(1). http://dx.doi .org/10.21503/log.Vil.1317

Bravo Salinas, G. A. y Vicente Acevedo, C. P. (2013). Liderazgo transformacional, engagement y desempeño intra-extra rol en un hospital público de la región del Maule (Tesis de grado). Universidad de Talca, Chile.

Cervera Cajo, L. (2012). Liderazgo transformacional del director y su relación con el clima organizacional en las instituciones educativas del distrito de Los Olivos (Tesis doctoral). Universidad Nacional Mayor de San Marcos, Lima, Perú.

Cesar Rivera, C. (2018). Liderazgo transformacional y desempeño directivo en la institución educativa $n^{\circ} 125$ "Ricardo Palma", San Juan de Lurigancho - 2017 (Tesis de maestría). Universidad César Vallejo, Perú.

Chacón Luna, G. (2016). Liderazgo transformacional y su relación con la satisfacción laboral (Tesis de licenciatura). Pontificia Universidad Católica del Perú, Lima, Perú.

Chen, J. (2013). The effect of kindergarten principals' leadership behaviors on teacher work performance. Social Behavior and Personality, 41(2), 251-262. https://doi.org/10.2224/ sbp.2013.41.2.251

Chiang Vega, M., Méndez Urra, G. y Sánchez Bernales, G. (2010). Cómo influye la satisfacción laboral sobre el desempeño: caso empresa de Retail. Theoria, 9(2), 21-36.

Chiavenato, I. (2002). Administración ( $3^{\mathrm{a}}$ ed.). Bogotá: McGraw Hill.

Coronado Quintana, J. A., Domínguez Canizales, K. G., Olivares Leal, A. y Retes López, R. (2014). Estilos de liderazgo de los agrónomos y su percepción de desempeño: caso Universidad de Sonora, México. Revista Mexicana de Agronegocios, 18(35), 1012- 1022.

Cruz Ortiz, V., Salanova Soria, M. y Martínez Martínez, I. (2013). Liderazgo transformacional y desempeño grupal: unidos por el engagement grupal. Revista de Psicología Social, 28(1), 183-196. Doi:10.1174/021347413806196762

Espinoza Poves, J. L. (2017). Clima organizacional y liderazgo: predictores del desempeño 


\section{EL LIDERAZGO DEL DIRECTOR Y EL DESEMPEÑO DOCENTE}

docente, en los centros educativos iniciales de la Unión Peruana del Norte, 2016 (Tesis doctoral). Universidad Peruana Unión, Lima, Perú.

Félix Román, J. J. (2014). Relación entre el liderazgo transformacional y el clima organizacional, en el instituto de educación superior tecnológico público Catalina Buendia de Pecho de Ica, durante el año 2013 (Tesis de maestría). Universidad Nacional de Educación Enrique Guzmán y Valle, Lima, Perú.

Fernández, M. C. y Quintero, N. (2017). Liderazgo transformacional y transaccional en emprendedores venezolanos. Revista Venezolana de Gerencia, 22(77), 56-74.

Fierro Ulloa, I. J. y Villalva, M. (2017). El liderazgo democrático, una aproximación conceptual. INNOVA, 2(4), 155-162.

García Rojas, V. C. (2017). Liderazgo transformacional directivo y desempeño docente en el centro preuniversitario de la Universidad $\mathrm{Na}$ cional de Educación "Enrique Guzmán y Valle” (Tesis de maestría). Universidad Nacional de Educación Enrique Guzmán y Valle, Lima, Perú.

Giraldo González, D. y Naranjo Agudelo, J. A. (2014). Liderazgo: desarrollo del concepto, evolución y tendencias (Estudio monográfico). Universidad del Rosario, Colombia.

González, O., González, O., Ríos, G. y León, L. (2013). Características del liderazgo transformacional presentes en un grupo de docentes universitario. TELOS, 15(3), 355-371.

Guanilo Pizarro, W. D. (2017). El liderazgo transaccional y su relación con el desempeño laboral en la empresa Conservas Ricofres, Chancay - 2017 (Tesis de maestría). Universidad César Vallejo, Perú.

Hermosilla, D., Amutio, A., Costa, S. y Páez, D. (2016). El liderazgo transformacional en las organizaciones: variables mediadoras y consecuencias a largo plazo. Journal of Work and Organizational Psychology, 32, 135-143. https://doi.org/10.1016/j.rpto.2016.06. 003

Huillca Condori, B. (2015). Liderazgo transformacional y desempeño docente en la especialidad de ciencias histórico - sociales del Instituto Pedagógico Nacional Monterrico (Tesis de maestría). Universidad Nacional Mayor de San Marcos, Lima, Perú.

Julca Chuquista, E. M. (2014). El liderazgo del equipo directivo y su relación con el desempeño docente, en la Asociación Educativa Adventista Sur Oriental del Perú, 2014 (Tesis de maestría). Universidad Peruana Unión, Lima, Perú.

Leal Soto, F., Albornoz Hernández, M., y Rojas Parada, M. (2016). Liderazgo directivo y condi- ciones para la innovación en escuelas chilenas: el que nada hace, nada teme. Estudios Pedagógicos (Valdivia), 42(2), 193-205. https://doi .org/10.4067/S0718-07052016000200011

Llorens, S., Salanova, M. y Losilla, J. (2009). Liderazgo transformacional y capital psicológico positivo: un estudio de caso en una empresa de construcción. Directivos Construcción, 220, 48-55.

Lowe, K. B., Kroeck, K. G. y Sivasubramaniam, N. (1996). Effectiveness of correlates of transformational and transactional leadership: A meta-analytic review of the MLQ literature. Leadership Quarterly, 7, 385-425. https://doi .org/10.1016/s1048-9843(96) 90027-2

Maldonado Yaranga, R. (2012). Percepción del desempeño docente en relación con el aprendizaje de los estudiantes (Tesis de grado). Universidad de San Martín de Porres, Perú.

Martínez de la Hidalga, Z. y Villardón Gallego, L. (2015). La imagen del profesor de educación secundaria en la formación inicial. Profesorado: Revista de Currículum y Formación de Profesorado, 19(1), 452-467.

Mendoza Torres, M. y Ortiz Riaga, C. (2006). El liderazgo transformacional, dimensiones e impacto en la cultura organizacional y eficacia de las empresas. Revista Facultad de Ciencias Económicas, 14(1), 118-134.

Minaya Canales, M. (2014). El liderazgo transformacional de los directivos y las actitudes de los docentes hacia el compromiso organizacional en la institución educativa $n^{\circ} 5084$ "Carlos Philips Previ” Callao 2010 - 2011 (Tesis de maestría).Universidad Nacional Mayor de San Marcos, Lima, Perú.

Mora Acuña. J. (2017). Liderazgo y desempeño laboral en docentes de instituciones educativas de San Martin de Porres, año 2016 (Tesis de maestría). Universidad César Vallejo, Perú.

Onorato, M. (2013). Transformational leadership style in the educational sector: an empirical study of corporate managers and educational leaders. Academy of Educational Leadership Journal, 17(1), 33-47.

Ortiz Chávez, A. (2016). Gestión de la calidad administrativa y su relación con el nivel de desempeño docente en la IE $N^{\circ} 0006$ “Aplicación” de Juanjui - 2016 (Tesis de maestría). Universidad César Vallejos, Perú.

Pacsi Choque, A.Y., Estrada Mejía, W., Pérez Vásquez, A. y Cruz Machaca, P. (2015). Liderazgo laissez faire. Revista Cuaderno Empresarial, 1(1), 9-16.

Parra Rivas, R. (2011). Liderazgo transformacional del director y desempeño laboral de los docentes. Revista Cientifica Digital del Centro 


\section{ORELLANA HERNÁNDEZ}

de Investigación y Estudios Gerenciales, 2(2), 54-72. Recuperado de http://www .grupocieg.org/archivos_revista/2-2-5\%20 (54-72)\%20Parra\%20Rosibel\%20rcieg\%20 noviembre\%2011_articulo_id70.pdf

Pazmiño Solys, G. A., Beltrán Morales, M. y Gallardo Media, W. M. (2016). Los estilos de liderazgo y su influencia en el desarrollo empresarial: caso pymes de la provincia de Tungurahua - Ecuador. PUCE, 103(1), 355-369.

Pedraja Rejas, L., Rodríguez Ponce, E., Barreda Olavarría, M., Sagredo Núñez, O. y Segovia León, C. (2009). Estilos de liderazgo y resultados del sistema de medición de la calidad de la educación: un estudio empírico en los colegios básicos de la ciudad de Arica-Chile. Revista Chilena de Ingeniería, 17(1), 21-26.

Pedraja Rejas, L., Rodríguez Ponce, E., Delgado Almonte, M. y Rodríguez Ponce, J. (2006). Liderazgo transformacional y transaccional: un estudio de su influencia en las pequeñas empresas. Ingeniare: Revista Chilena de Ingeniería, 14(2), 159-166. http://dx.doi.Org/10.4067/ S0718-330522006000100010

Pedraja Rejas, L., Rodríguez Ponce, E. y Rodríguez Mardones, P. (2016). Estilos de liderazgo de dirección escolar y el logro académico de los estudiantes: un estudio exploratorio. Interciencia, 41(11), 748-756.

Pérez Perea, L., Soler Cárdenas, S. F. y Díaz Hernández, L. (2009). Ambiente laboral en los policlínicos universitarios. Educación Médica Superior, 23(2). Recuperado de http://scielo .sld.cu/pdf/ems/v23n2/ems04209.pdf

Perilla Toro, L. y Gómez Ortíz, V. (2017). Relación del estilo de liderazgo transformacional con la salud y el bienestar del empleado: el rol mediador de la confianza en el líder. Revista de Psicología del Trabajo y de las Organizaciones, 33(2), 95-108.

Ponce Luque, E. J. (2018). Liderazgo directivo y desempeño docente en la institución educativa Manuel Veramendi e Hidalgo del distrito de Mariano Melgar, Arequipa 2017 (Tesis de maestría). Universidad Nacional de San Agustín Arequipa, Perú.

Ponce Vidal, R. A. (2008). El liderazgo y su relación con el rendimiento académico (Tesis de maestría). Universidad del Bío Bío, Chile.

Quispe Quispe, P. (2011). Relación entre el estilo de liderazgo del director y el desempeño docente en las instituciones educativas públicas del 2 do. Sector de Villa El Salvador de la UGEL 01 San Juan de Miraflores, en los años 2009 y 2010 (Tesis de maestría). Universidad Nacional Mayor de San Marcos, Lima, Perú.

Ramón Molina, D. G., Muñoz Aparicio, C. G., An- cona Alcocer, M. C. y Navarrete Torres, M. C. (2015). El liderazgo y su influencia en el aprendizaje en el estudiante de Mercadotecnia. Revista Internacional de Organización Educativa $y$ Liderazgo, 2(2), 65-74.

Ramos Cuba, J. J. (2015). Liderazgo del director y el desempeño docente en las I.E. de primaria de la red $n^{\circ} 03$ de la Ugel $n^{\circ} 02$ del Rímac, Lima, año 2012 (Tesis de maestría). Universidad Peruana Unión, Perú.

Reyes Gastañadui, N. H. (2018). Liderazgo transaccional y transformacional con el desempeño laboral según el proceso de atención de enfermería en los profesionales de enfermería estudiantes de las especialidades en la unidad de posgrado UpeU Lima - 2017 (Tesis de maestría). Universidad Peruana Unión, Perú.

Reynaga Utani, Y. (2015). Motivación y desempeño laboral del personal en el hospital Hugo Pesce Pescetto de Andahuaylas, 2015 (Tesis de grado). Universidad Nacional José María Arguedas, Perú.

Rodríguez Ponce, E., Pedraja Rejas, L. y Ganga Contreras, F. (2017). La relación entre los estilos de liderazgo y el desempeño de los equipos de dirección intermedia: un estudio exploratorio desde Chile. Contabilidad y Negocios, 12(23), 129-144.

Rojas Jara, A. (2012). El liderazgo transformacional en directores de tres liceos bicentenario y tres $l i$ ceos regulares de la región metropolitana (Tesis de maestría). Universidad de Chile, Chile.

Salas Vallina, A. (2013). Liderazgo transformacional, capacidad de aprendizaje organizativo y felicidad en el trabajo (Tesis doctoral). Universidad de Valencia, Valencia.

Salem, H. (2015). The impact of leadership styles on job satisfaction and mediating role of perceived organizational politics. Procedia- Social and Behavioral Sciences, 172, 563-569. https://doi.org/10.1016/j.sbspro.2015.01.403

Sgreccia, N. y Cirelli, M. (2015). Cualidades de docentes memorables destacadas por aspirantes a profesor en matemática. Profesorado Revista de Curriculum y Formación de Profesorado, 19(2), 333-350.

Silva Peralta, Y., Olsen, C., Pezzi, L. y Sanjurjo, N. (2016). Liderazgo transaccional y transformacional de voluntarios jóvenes y adultos de Mar del Plata. Psicoperspectivas, 15(3), 146-157.

Sum Mazariegos, M. (2015). Motivación y desempeño laboral (Tesis de grado).Universidad Rafael Landívar, Guatemala.

Yang, M. (2012). Transformational leadership and Taiwanese public relations practitioners' job satisfaction and organizational commitment. Social Behavior and Personality, 40(1), 31-46. https://doi.org/10.2224/sbp.2012.40.1.31 


\section{EL LIDERAZGO DEL DIRECTOR Y EL DESEMPEÑO DOCENTE}

Zarate Ramírez, D. (2011). Liderazgo directivo y el desempeño docente en instituciones educativas de primaria del distrito de Independencia (Tesis de maestría). Universidad Nacional Mayor de San Marcos, Lima, Perú.
Recibido: 22 de noviembre de 2018

Revisado: 15 de diciembre de 2018

Aceptado: 11 de enero de 2019 